OPEN

SUBJECT AREAS:

SINGLE PHOTONS AND

QUANTUM EFFECTS

NANOWIRES

QUANTUM OPTICS

OPTICAL SENSORS

Received

18 November 2014

Accepted

9 March 2015

Published

15 April 2015

Correspondence and requests for materials should be addressed to

L.X.Y. (lxyou@mail. sim.ac.cn)

\section{Single photon detector with high polarization sensitivity}

\author{
Qi Guo, Hao Li, LiXing You, WeiJun Zhang, Lu Zhang, Zhen Wang, XiaoMing Xie \& Ming Qi
}

State Key Laboratory of Functional Materials for Informatics, Shanghai Institute of Microsystem and Information Technology (SIMIT), Chinese Academy of Sciences, 865 Changning Rd., Shanghai 200050, China.

Polarization is one of the key parameters of light. Most optical detectors are intensity detectors that are insensitive to the polarization of light. A superconducting nanowire single photon detector (SNSPD) is naturally sensitive to polarization due to its nanowire structure. Previous studies focused on producing a polarization-insensitive SNSPD. In this study, by adjusting the width and pitch of the nanowire, we systematically investigate the preparation of an SNSPD with high polarization sensitivity. Subsequently, an SNSPD with a system detection efficiency of $12 \%$ and a polarization extinction ratio of 22 was successfully prepared.

P olarization, together with amplitude, phase and frequency or wavelength, are the four fundamental properties of light. Understanding and utilizing polarization could greatly expand and enrich optical applications. Numerous applications based on the detection of polarization have been explored, such as polarization-coding quantum key distribution ${ }^{1}$, biomedical applications ${ }^{2,3}$, remote sensing ${ }^{4}$, and polarization imaging $^{5}$. Nevertheless, most conventional optical detectors are intensity detectors, which are insensitive to the polarization of light. To achieve polarization-sensitive detection, an additional polarizing filter or stand-alone polarizer is necessary before the optical detector ${ }^{6}$. Advanced ultrasensitive optical detection has approached the epoch of single photon detection. However, there are no reports on semiconducting single photon detectors (SPDs) with polarization sensitivity to the best of our knowledge.

In the last decade, a novel SPD based on a superconducting meandered nanowire was demonstrated to exhibit better performance (high detection efficiency, low dark count rate, high repetition rate, and small timing jitter) beyond that of the traditional semiconducting SPDs for near infrared wavelengths ${ }^{7-11}$. The detection mechanism of the superconducting nanowire single photon detector (SNSPD) is the generation and disappearance of a photon-induced resistive domain across the superconducting nanowire upon absorptance of a photon ${ }^{7}$. Due to its unique anisotropic geometric structure, an SNSPD is naturally polarization sensitive ${ }^{12}$. However, most previous studies focused on producing a polarization-insensitive SNSPD ${ }^{8,12-14}$.

In this paper, we present our recent work on fabricating an SPD with high polarization sensitivity. We systematically discuss the relationships between the polarization extinction ratio (PER) and SNSPD geometric parameters (width, pitch, etc.). SNSPDs with high PER are fabricated, and a detection efficiency (DE) of $12 \%$ and PER of 22 are successfully attained.

\section{Results}

Model and analysis. The polarization dependence of SNSPDs originates from their anisotropic meandered nanowire geometry. For a transverse magnetic (TM) wave with the electrical field perpendicular to the nanowire, the magnetic field intensity is uniform across the wire; for a transverse electric (TE) wave with the magnetic field perpendicular to the nanowire, the electrical field intensity is uniform across the wire. When the detector is illuminated from either the top or the bottom through the substrate by a TM wave, the generated charges at the edge of the nanowire result in discontinuities of the electric field, which screen the nanowire and reduce the electric field intensity inside of the nanowire leading to decreased absorptance. However, for a TE wave, no discontinuities appear at the edge of the nanowire, and the electric field is relatively evenly distributed across the wire, which results in a higher absorptance. Therefore, the absorptance difference of the nanowire for TM and TE waves leads to the polarization sensitivity of the SNSPD. In general, to achieve high polarization sensitivity, the nanowire should be narrow and with a smaller filling ratio.

To quantitatively investigate the polarization sensitivity of the SNSPD, we performed numerical simulations using the finite element method (FEM). The physical picture is the same for both front- and back-side illumina- 

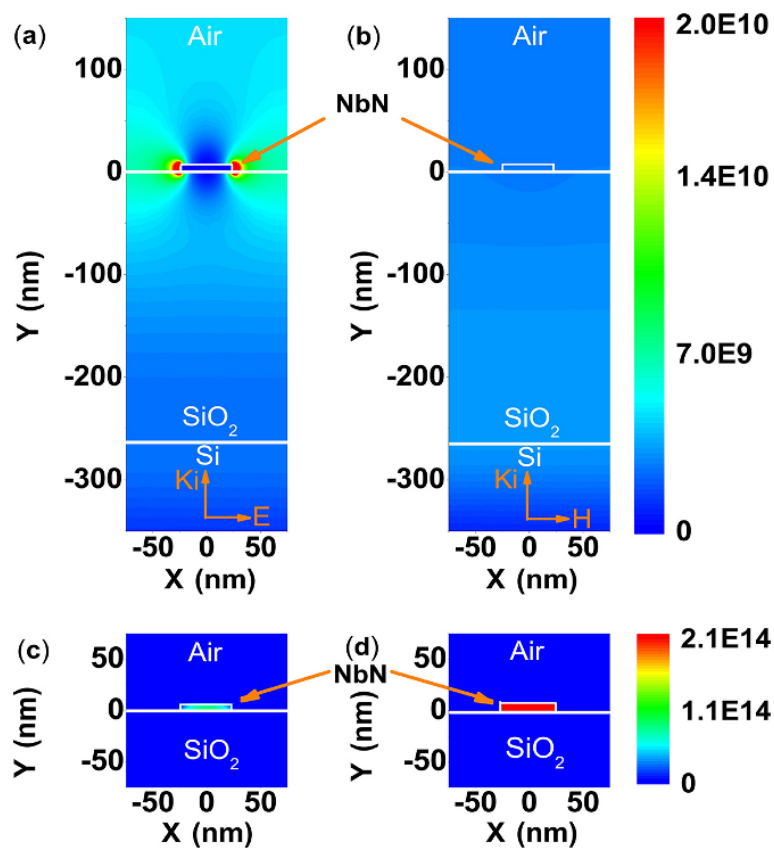

Figure 1 Simulation results of SNSPD. (a) \& (b) Electric field intensity distribution when TM (a) and TE (b) waves are incident on the nanowire from the back of the substrate. (c) \& (d) Electromagnetic power loss density distribution for TM (c) and TE (d) waves.
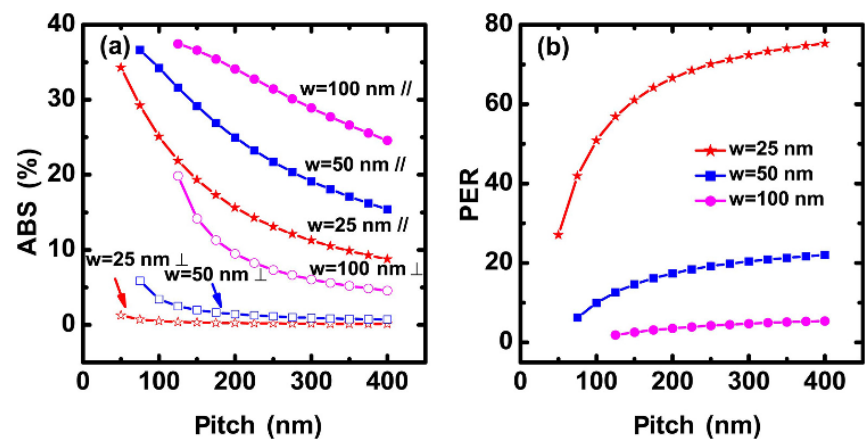

Figure $2 \mid$ Calculation of ABS and PER. (a) Numerical results of the absorptance (ABS) of $\mathrm{NbN}$ nanowires with a thickness of $7 \mathrm{~nm}$ as a function of pitch for various widths, TE $(/ /)$ and TM $(\perp)$ waves. (b) Calculated PER versus pitch for various linewidths using the data in (a). tion. Backside illumination is selected here for the possibility of integrating an optical cavity on the top in the future. With Comsol Multiphysics, under RF module, a model is set up as shown in Figs. 1 (a) and (b) for one unit cell of the grating, flanked by Floquet boundary conditions describing the periodicity. Port conditions are used both for specifying the incident wave and for letting the resulting solution leave the model without any non-physical reflections. This simulation is equivalent to an infinitely extended periodic structure in the horizontal direction and therefore neglects any edge effects of the real meander ${ }^{15-17}$. The estimated accuracy of the simulations is $10^{-3}$, which is enough for the evaluation of the design and the comparison with the experimental results. All the simulations and measurements were performed for a $1550 \mathrm{~nm}$ wavelength. Figs. 1(a) and (b) show the electric field intensity for the TM wave and TE wave, respectively. The thickness $t$ and width $w$ of the superconducting $\mathrm{NbN}$ nanowire were selected to be $7 \mathrm{~nm}$ and $50 \mathrm{~nm}$, respectively, with a pitch $p$ of $100 \mathrm{~nm}$, which are reasonable geometric values for fabrication. The refractive indices of $\mathrm{NbN}, \mathrm{Si}$ and $\mathrm{SiO}_{2}$ were considered to be $5.23-5.82 \mathrm{i}, 3.45$ and 1.45 , respectively ${ }^{15,16}$. The thickness of $\mathrm{SiO}_{2}$ layer was a quarter of the wavelength. The energy of the electric field spills out of the nanowire in Fig. 1(a) because of the charges induced by the electrical field perpendicular to the nanowire. However, a relatively uniform electric field appears across the nanowire for the TE wave in Fig. 1(b). Given the field distribution, the absorptance (ABS) was determined by:

$$
A B S=\frac{\iint_{\Omega} \frac{1}{2} \omega \operatorname{Im}\left[\varepsilon_{1}\right]\left|E_{1}\right|^{2} d x d y}{Q_{0}}
$$

where $\omega$ is the angular frequency of the incident wave, $Q_{0}$ is the incident power, $\Omega$ is the cross section of the nanowire, $\varepsilon_{1}$ is the permittivity of the superconducting nanowire and $E_{1}$ is the internal electric field of the nanowire. To more intuitively illustrate the absorptance, the electromagnetic power loss densities (absorptance) in the nanowire are presented in Figs. 1(c) and (d) for TM and TE waves, respectively, which indicate that the absorptance for the TM wave is effectively smaller than that of the TE wave. In other words, SNSPDs may exhibit strong polarization sensitivity.

Fig. 2(a) shows the calculated absorptance dependence on the pitch for TM and TE waves. Three representative widths $(25,50$, and $100 \mathrm{~nm}$ ) of the nanowire were selected for comparison. A thickness of $7 \mathrm{~nm}$ was adopted for the calculation, which is a reasonable value for a high-performance SNSPD fabricated on a Si substrate. In principle, a high filling ratio (large width and small pitch) corresponds to a high absorptance for both TM and TE waves. However, the enhancement of absorptance for the TM wave is stronger than that for the TE wave. The corresponding polarization
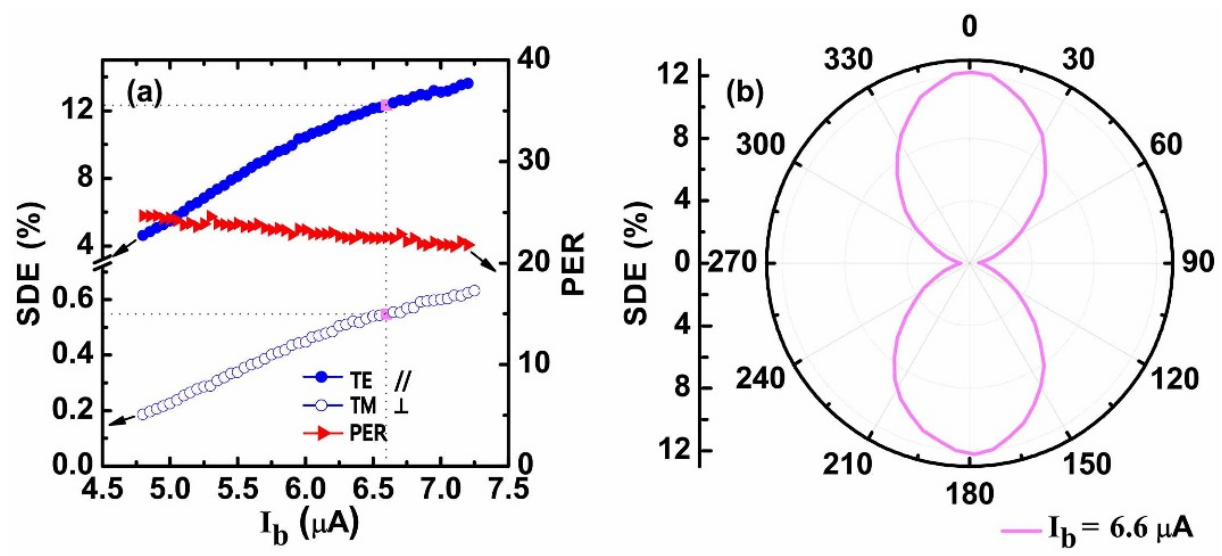

Figure $3 \mid$ Measurement results of SNSPD. (a) SDE and PER as functions of bias current. The blue solid and hollow dots represent the SDE for TE and TM waves, respectively. The PER, represented by the red triangles, is calculated from the SDE data for two different polarizations. (b) The polarization dependence of SDE in the polar coordinate system at a dark count rate of $100 \mathrm{~Hz}$. 

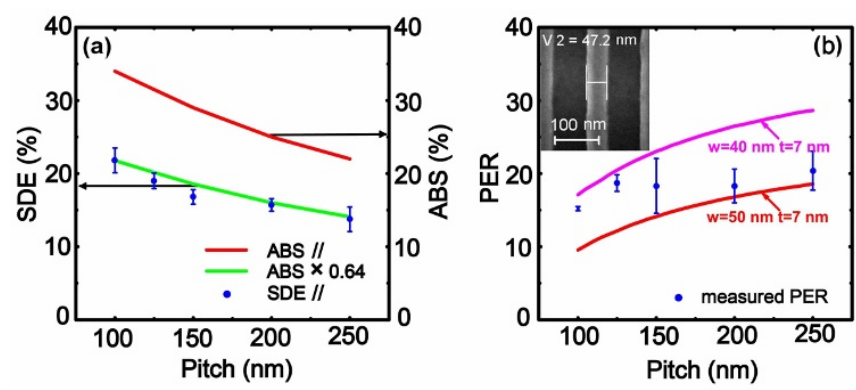

Figure $4 \mid$ Comparison and analysis of 16 SNSPDs with different pitches. (a) Plot of SDE and ABS for the TE wave as a function of the pitch. The blue dots represent the measured SDEs of the devices. The error bar indicates the standard deviation of the SDEs for SNSPDs with the same pitch. The red line represents the calculated absorptance, and the green line shows the estimated SDE from the absorptance curve considering a loss factor of 0.64. (b) Comparison of the measured PERs (blue scattered dots) with the calculated PER as a function of pitch. The red and pink lines represent linewidths of 50 and $40 \mathrm{~nm}$, respectively. The inset presents an SEM image of a typical nanowire, revealing a linewidth of $47.2 \mathrm{~nm}$. The error bar indicates the standard deviation of the PERs for SNSPDs with the same pitch.

extinction ratio (PER) for the SNSPD is shown in Fig. 2(b). Here the PER is defined as the absorptance ratio of the TE to TM wave. Fig. 2 (b) demonstrates that a smaller width and a larger pitch result in a larger PER. For example, the PER may reach 70 for a SNSPD with parameters of $w=25 \mathrm{~nm}$ and $p=250 \mathrm{~nm}$. However, the corresponding maximal absorptance for the TE wave is only $12 \%$. Therefore, there is a tradeoff for a SNSPD with a high PER and an acceptable DE. In addition, the process complexity of the device fabrication should also be considered.

Experimental results. SNSPD devices were fabricated from NbN film deposited on thermal oxidized Si substrate and packaged with light illuminated from the backside of the substrate. The devices were cooled down to $2.3 \mathrm{~K}$ for the characterization. Detailed information concerning device preparation, characterization and measurement setup can be found in the methods' section below. Fig. 3(a) shows the current dependence of the system detection efficiency (SDE) for parallel and vertical polarizations as well as the PER for a $50 \mathrm{~nm}$ linewidth and $250 \mathrm{~nm}$-pitch device. The SDE for the parallel polarization is one order of magnitude higher than the SDE for vertical polarization, which yields a PER range of $21-25$ for the entire bias range. The slight decrease of PER in the bias range was unclear at this point. At the dark count rate of $100 \mathrm{~Hz}$ marked with pink dots, we obtained a PER of 22 with a maximal SDE of $12 \%$. By tuning the state of polarization (SOP), the polarization dependence of the SDE was measured and is displayed in Fig. 3(b) when the device was biased at a current of $6.6 \mu \mathrm{A}$ with a dark count rate of $100 \mathrm{~Hz}$. The "peanut" curve further demonstrates the strong polarization sensitivity of the SNSPD.

To study the pitch dependence of SDE and PER, 16 SNSPDs with 5 different pitch values $(100,125,150,200$, and $250 \mathrm{~nm})$ were measured. The blue dots in Fig. 4(a) represent the measured maximal SDEs of SNSPDs for TE wave with different pitches in the same measurement condition. The measured maximal SDEs are smaller than the calculated ABS for the TE wave represented by the red line. However, considering the existing system optical loss and non-unity intrinsic detection efficiency, a factor may be adopted to reexamine the relation between ABS and SDE. Represented by the green curve in Fig. 4(a), the measured SDE matches with the absorptance with a factor of 0.64. Furthermore, the PERs for SNSPDs with different pitches were calculated according to the measured SDE $(/ /)$ and SDE $(\perp)$ and are shown in Fig. 4(b), which deviate from the calculated values (red curves in Fig. 4(b)). This finding may be explained by the discrepancy of the width of the nanowire from the designed value, though other issues like the film thickness may also have contribution to it. The calculation results in Fig. 2(b) indicate that PER is very sensitive to the linewidth of the nanowire especially when the linewidth is smaller. One typical scanning electron microscopic (SEM) image of the nanowire shown as the inset of Fig. 4(b) reveals that the real linewidth may be slightly smaller than $50 \mathrm{~nm}$. A smaller linewidth of $40 \mathrm{~nm}$ results in a higher PER, as represented by the pink line in Fig. 4(b). The measured PER values are almost all located between the two curves for the $50 \mathrm{~nm}$ and $40-\mathrm{nm}$ widths.

\section{Discussion}

We have demonstrated that an SNSPD with a PER over 20 and SDE of $12 \%$ can be fabricated. By improving the optical coupling and material optimization, the SDE can be further increased to the maximal absorptance $(>20 \%)$. In future studies, the width of the nanowire should be decreased in order to increase the PER. However, there will be some process challenges such as those involved with the electron beam lithography. The use of the popular optical cavity structure may effectively improve the SDE by increasing ABS. However, the PER decreases because the absorptance for a TM wave increases much more than that for a TE wave. Therefore, it is necessary to develop a new polarization-selective optical structure to

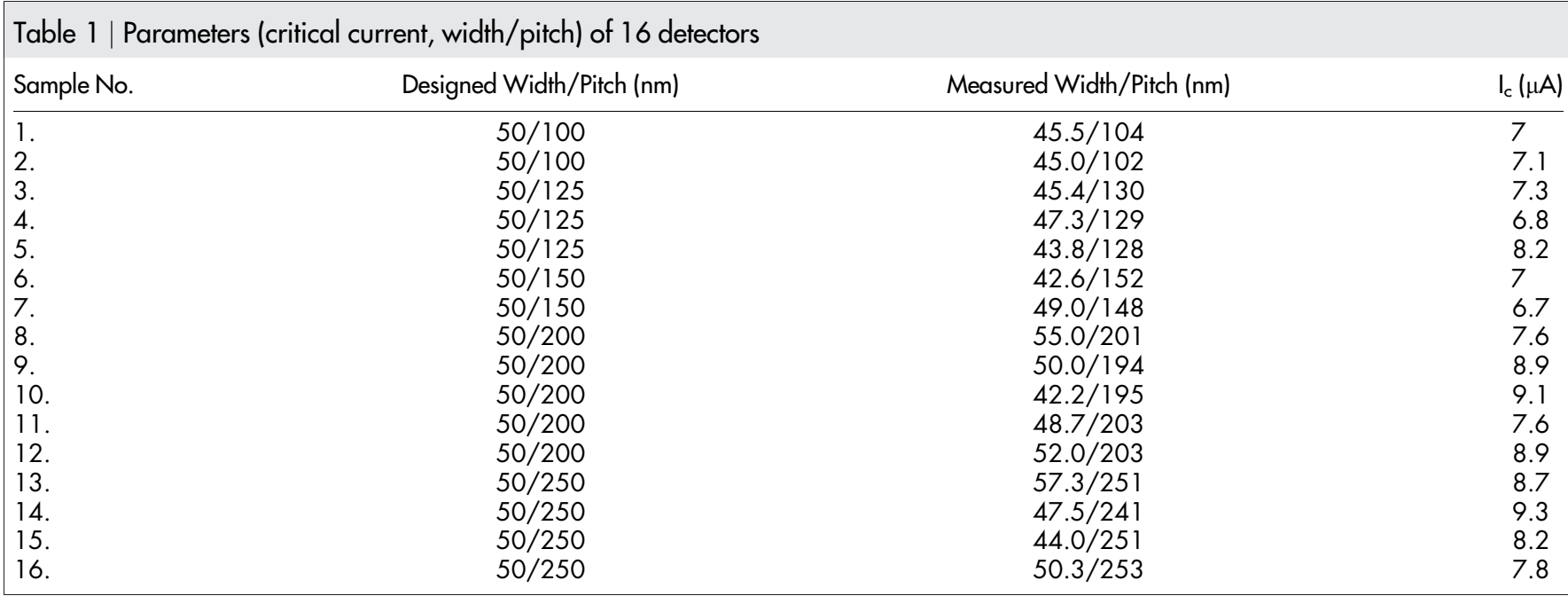




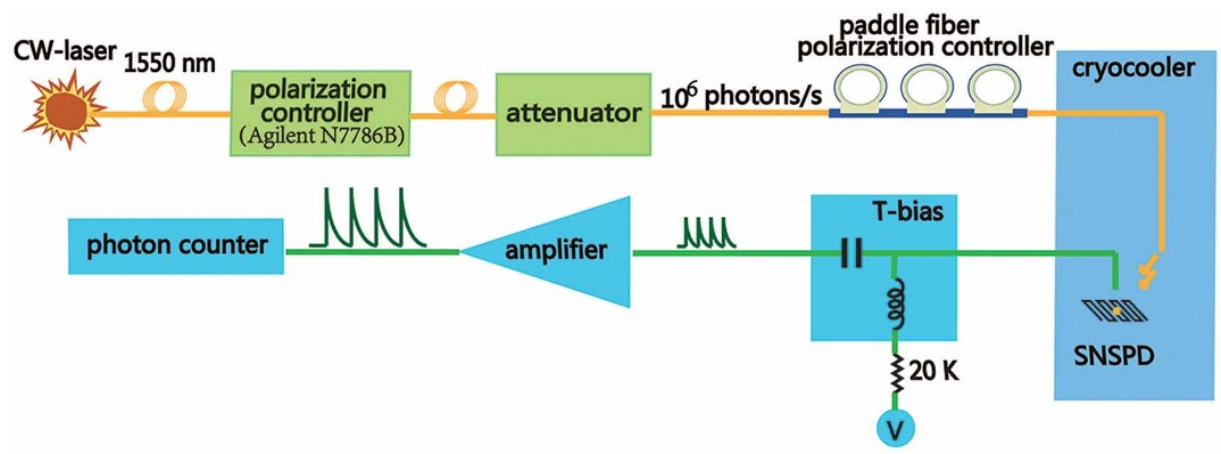

Figure $5 \mid$ Schematics of the measurement system. Optical (represented yellow line) and electric (represented by green line) components are shown in the figure.

increase both the DE and PER in the future, like integrated plasmonic structures proposed by Csete et al. ${ }^{18,19}$.

In summary, an approach to produce a single photon detector with high polarization sensitivity was reported. By fabricating an SNSPD with a width of $50 \mathrm{~nm}$ and pitch of $250 \mathrm{~nm}$, an SNSPD with a SDE of $12 \%$ and a PER of 22 was produced. The SDE and PER dependence on the width and pitch were systematically studied using both simulations and experiments, and the results match well with each other. We believe that there is much room for improving both PER and SDE. The SNSPD with high PER provides a unique solution for single photon counting applications based on polarization sensitivity, such as polarization imaging.

\section{Methods}

Device preparation. $\mathrm{NbN}$ thin film with a thickness of $7 \mathrm{~nm}$ was deposited on a double-side oxidized silicon substrate at room temperature using reactive DC magnetron sputtering in a mixture of $\mathrm{Ar}$ and $\mathrm{N}_{2}$ gases. The film was patterned into a meandered nanowire structure by electron beam lithography using a positive-tone Polymethyl Methacrylate electron-beam resist and was reactively etched in $\mathrm{CF}_{4}$ plasma. The nanowire covers an active area of $15 \times 15 \mu^{2}$ with a linewidth of $50 \mathrm{~nm}$, which is easier to fabricate and control than a $25 \mathrm{~nm}$-wide nanowire. Five different pitches $(100,125,150,200$, and $250 \mathrm{~nm})$ were selected for five different types of SNSPDs on the same wafer. Each wafer includes 25 devices and each type has 5 samples. A $50-\Omega$-matched coplanar transmission line was formed using ultraviolet lithography and reactive ion etching. A lensed optical fiber was vertically aligned to the sensitive area from the backside of the substrate using a copper package-box. The distance between the fiber tip and substrate was controlled around $20 \pm 5 \mu \mathrm{m}$ to ensure good optical focus. The beam waist of the focused light-spot is about $10 \mu \mathrm{m}$. Then, the SNSPDs were mounted in a custom-designed 6-channel cryostat based on a compact Gifford-McMahon cryocooler and cooled to $2.3 \mathrm{~K}$. All the 25 detectors on the same chip with five different geometric parameters were measured. Due to the existence of possible defects or the imperfect fabrication process, 9 detectors had suppressed critical currents $(<5.0 \mu \mathrm{A})$ and the results were discarded. All the other 16 SNSPDs had critical currents between $6.7 \mu \mathrm{A}$ and $9.3 \mu \mathrm{A}$. The critical currents and the corresponding width/pitch of the 16 detectors are shown in Table 1 . Indeed it is difficult to give the precise widths based on the SEM measurement, and the variation of the linewidth exists even for one nanowire. Besides, multiple measurement by SEM may damage the detector. The variation of $I_{c}$ is related to the possible defects in or the different width/thickness of the nanowire. We discussed the data of the 16 SNSPDs and there are at least two detectors for each pitch.

Measurement setup. Schematics of the optical and electrical setups are shown in Fig. 5. A fiber-coupled continuous-wave tunable laser source (Agilent 81980A) was used as the photon source. A 2-port polarization controller (Agilent N7786B) was adopted to provide photons with any linear polarization. Due to the minimal power limitation $(-10 \mathrm{dBm})$ of Agilent N7786B, three attenuators were adopted after N7786B, which provided the light intensity to a level of $10^{6}$ photons/s. Since the attenuators and the fibers connected may also change the SOP of the photons, a paddle fiber polarization controller was inserted to further adjust the SOP of photons arriving at the device. When the SOP of photons was optimized, the paddle fiber polarization controller was fixed. Then the SOP was precisely adjusted by Agilent N7786B. For the electrical characterization, an isolated voltage source in series with a $20 \mathrm{k} \Omega$ resister was adopted as a quasi-constant-current source for biasing. Voltage pulses arising from SNSPD were amplified using a $50 \mathrm{~dB}$ low noise amplifier (RF Bay Inc. LNA-650) and discriminated by a $200 \mathrm{MHz}$ frequency photon counter (ThinkSRS SR400). All the electronics and optics were operated at room temperature except the fibers and coaxial cables were connected from room temperature to low temperature SNSPDs.

1. Gisin, N., Ribordy, G. G., Tittel, W. \& Zbinden, H. Quantum cryptography. Rev Mod. Phys. 74, 145-195 (2002).

2. Gramatikov, B. I., Zalloum, O. H. Y., Wu, Y. K., Hunter, D. G. \& Guyton, D. L. Directional eye fixation sensor using birefringence-based. Appl. Opt. 46, 1809-1818 (2007).

3. Salomatina-Motts, E., Neel, V. A. \& Yaroslavskaya, A. N. Multimodal Polarization System for Imaging Skin Cancer. Opt. Spectrosc. 107, 884-890 (2009).

4. Tyo, J. S., Goldstein, D. L., Chenault, D. B. \& Shaw, J. A. Review of passive imaging polarimetry for remote sensing applications. Appl. Opt. 45, 5453-5469 (2006).

5. Treibitz, T. \& Schechner, Y. Y. Active Polarization Descattering. Ieee T Pattern Anal 31, 385-399 (2009).

6. Sanshiro, S., Toshihiko, N., Kiyotaka, S., Takashi, T. \& Jun, O. Polarization Analyzing Image Sensor with On-Chip Metal Wire Grid Polarizer in 65-nm Standard Complementary Metal Oxide Semiconductor Process. Jpn. J. Appl. Phys. 50, 04DL01 (2011)

7. Natarajan, C. M., Tanner, M. G. \& Hadfield, R. H. Superconducting nanowire single-photon detectors: physics and applications. Supercond. Sci. Technol. 25, 063001 (2012).

8. Marsili, F. et al. Detecting single infrared photons with $93 \%$ system efficiency. Nat. Photonics 7, 210-214 (2013).

9. Yamashita, T., Miki, S., Terai, H. \& Wang, Z. Low-filling-factor superconducting single photon detector with high system detection efficiency. Opt. Express 21, 27177-27184 (2013).

10. Yang, X. et al. Superconducting nanowire single photon detector with on-chip bandpass filter. Opt. Express 22, 16267-16272 (2014).

11. You, L. et al. Jitter analysis of a superconducting nanowire single photon detector. AIP Advances 3, 072135 (2013).

12. Dorenbos, S. N. et al. Superconducting single photon detectors with minimized polarization dependence. Appl. Phys. Lett. 93, 161102 (2008).

13. Verma, V. B. et al. A three-dimensional, polarization-insensitive superconducting nanowire avalanche photodetector. Appl. Phys. Lett. 101, 251114 (2012).

14. Driessen, E. F. C. et al. Impedance model for the polarization-dependent optical absorption of superconducting single-photon detectors. Eur Phys J-Appl Phys 47, (2009).

15. Anant, V. et al. Optical properties of superconducting nanowire single-photon detectors. Opt. Express 16, 10750-10761 (2008).

16. Li, H. et al. Nonideal Optical Cavity Structure of Superconducting Nanowire Single-Photon Detector. IEEE Journal of Selected Topics in Quantum Electronics 20, 3803705 (2014)

17. Hu, X., Dauler, E. A., Molnar, R. J. \& Berggren, K. K. Superconducting nanowire single-photon detectors integrated with optical nano-antennae. Opt. Express 19, 17-31 (2011).

18. Csete, M. et al. Improvement of infrared single-photon detectors absorptance by integrated plasmonic structures. Sci. Rep. 3, 2406 (2013).

19. Csete, M., Szekeres, G., Szenes, A., Szalai, A. \& Szabo, G. Plasmonic structure integrated single-photon detector configurations to improve absorptance and polarization contrast. arXiv preprint arXiv: 1409.4720 (2014).

\section{Acknowledgments}

This work was funded by Strategic Priority Research Program (B) of the Chinese Academy of Sciences (XDB04010200\&XDB04020100) and partially supported by the National Natural Science Foundation of China $(91121022,61401441,61401443)$ and National Basic Research Program of China (2011CBA00202). 


\section{Author contributions}

Q.G. and H.L. preformed COMSOL calculation and analysed the data. L.Z. provided the $\mathrm{NbN}$ film. Q.G. and W.J.Z. prepared the samples and designed and performed the experiments. Q.G. wrote the paper. L.X.Y. proposed the original idea and organized the research. Z.W., X.M.X. and M.Q. discussed the results. All authors reviewed the manuscript.

\section{Additional information}

Competing financial interests: The authors declare no competing financial interests.
How to cite this article: Guo, Q. et al. Single photon detector with high polarization sensitivity. Sci. Rep. 5, 9616; DOI:10.1038/srep09616 (2015)

(i) This work is licensed under a Creative Commons Attribution 4.0 International License. The images or other third party material in this article are included in the article's Creative Commons license, unless indicated otherwise in the credit line; if the material is not included under the Creative Commons license, users will need to obtain permission from the license holder in order to reproduce the material. To view a copy of this license, visit http://creativecommons.org/licenses/by/4.0/ 